\title{
Determination of Pore Radius Distribution of Porous Polymeric Membranes by Electron Microscopic Method
}

\author{
Sei-ichi Manabe, Yukari Shigemoto, and Kenji Kamide \\ Textile Research Laboratory, Asahi Chemical Industry Co., Ltd., \\ Takatsuki, Osaka 569, Japan
}

(Received November 12, 1984)

\begin{abstract}
An attempt was made to establish a method for evaluating the pore characteristics of porous polymeric membrane having the mean pore diameter larger than $10 \mathrm{~nm}$ through electron micrograph with help of stereology (EM method). For this purpose, ellipsoidal, spherical, and straight-through cylindrical pore models were adopted. Theoretical equations relating the pore radius distribution function $N(r)$ to the distribution function $F(x)$ of the length $x$ of test lines cut off by pores in an electron micrograph were derived as follows;
\end{abstract}

$$
\begin{array}{ll}
N_{\mathrm{e}}(r)=-\frac{4}{\pi \Phi_{\mathrm{e}}}\{\mathrm{d}(F(x) / x) / \mathrm{d} x\} & \text { (for ellipsoidal pore) } \\
N_{\mathrm{s}}(r)=-\frac{4}{\pi}\{\mathrm{d}(F(x) / x) / \mathrm{d} x\} & \text { (for spherical pore) } \\
F(x)=x \int_{x / 2}^{\infty} \frac{N(r)}{\left(4 r^{2}-x^{2}\right)^{1 / 2}} \mathrm{~d} r & \text { (for straight-through cylindrical pore) }
\end{array}
$$

where, $\Phi_{\mathrm{e}}$ is a shape factor and the suffix e and s denote ellipsoidal and spherical pores. The mean pore radii, porosity, and pore radius distribution functions were evaluated from $N(r)$ and compared with those determined by various methods including mercury intrusion, fluid permeability and bubble pressure, flow rate, surface area, apparent density, and swelling methods for porous regenerated cellulose membrane prepared by micro-phase separation method and polycarbonate membrane Nuclepore. ${ }^{\circledR}$

KEY WORDS Pore Characteristics / Porous Polymeric Membrane / Pore Radius Distribution / Mean Pore Radius / Porosity / Regenerated Cellulose / Ellipsoidal Pore / Spherical Pore / Cylindrical Pore / Nuclepore ${ }^{\circledR}$ / Electron Microscopy /

A variety of performance of porous polymeric membrane is predominantly governed by its pore characteristics, especially the pore radius distribution, from which numerous parameters characterizing the pore can be derived. ${ }^{1}$ The determination of the pore radius distribution is paramountly important in designing the performance and quality control of a membrane and can be carried out by various methods, such as methods of electron microscope (EM), mercury intrusion (MI), fluid permeability and bubble pressure (BP), ultrafiltration-permselectivity (UP), and gas permeability (GP). ${ }^{2}$ All of these methods, except for EM method, need an assumption on the pore shape. In contrast, EM method gives us the direct information on the shape and radius of pores existing in the membrane. Scanning electron microscopic (SEM) method is applicable only to the membranes with pore diameter larger than $c a$. $10 \mathrm{~nm}$, because of lower resolving power of SEM. ${ }^{2}$ When the pore diameter is less than $10 \mathrm{~nm}$ and probably larger than $1 \mathrm{~nm}$, we can apply transmission 
electron microscopic (TEM) method, instead of SEM method, to the ultra-thin section, with ca. $100 \mathrm{~nm}$ thick, the membrane swollen with a solvent ${ }^{3}$ or stained with a heavy metal. ${ }^{4}$

It should be noticed that all the pore radius distributions estimated hitherto using EM method $^{5,6}$ are derived assuming that the pore radius distribution on the surface of a membrane is same even at inner part of the membrane and that the shape of pores is circular. In other words, these distributions are, in the strict sense, reliable only in the case of straightthrough cylindrical pores. This assumption is not generally acceptable for the actual membranes and therefore a more reasonable and accurate EM method is highly anticipated.

In this study we intend to propose a sophisticated EM method for evaluation of the pore characteristics with help of stereology ${ }^{7}$ and to clarify the feature of the new EM method in comparison with other methods, such as MI, BP, surface area (SA), flow rate (FR), apparent density, and swelling methods. For this purpose, we adapt the model membrane with straight-through cylindrical pores and that with pores of complicated shape.

\section{THEORETICAL BACKGROUND}

The pore on the membrane can be classified from the view point of the state of its connection into three types: isolated, semi-open, and open pores. In this paper, the shape of pores on any sectional plane parallel to the membrane surface is assumed to be circular, though most of actual membranes have pores with non-circular shape.

Consider six typical pore models different in pore shape and in pore dispersion as demonstrated in Figure 1. The straight-through cylindrical pore in Figure 1(a) is the simplest and ideal pore model to explain fluid permeation phenomena of the membrane and is actually observed in a polycarbonate membrane, Nuclepore. ${ }^{\circledR}$ The truncated cone shape pore model in Figure 1(b) was proposed by

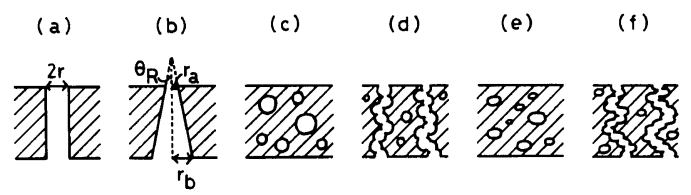

Figure 1. Schematic representation of six different pore models: (a), straight-through cylindrical pore; (b), truncated cone shape pore; (c), spherical isolated pore; (d), spherical open pore; (e), ellipsoidal isolated pore; (f), ellipsoidal open pore; $r$, radius of pore $\left(r_{\mathrm{a}}\right.$, front side; $r_{\mathrm{b}}$, back side).

$\mathrm{us}^{8}$ in order to take into consideration the difference in the pore diameter between front and back surfaces of a membrane. The spherical and/or ellipsoidal models of Figure 1(c), (d), (e), and (f) are applicable to the membrane prepared through the micro-phase separation method. ${ }^{9}$ The open pore may be generated by the collision of many pore particles. ${ }^{10}$ The shape of this open pore can be approximated by the shape of the continuous row of the pore particle. That is, the open spherical pore model in Figure 1(d) is regarded as the special case of the spherical pore model in Figure 1(c), and also, the open ellipsoidal pore model in Figure 1(f) as that of the ellipsoidal pore model in Figure 1(e).

\section{Ellipsoidal Pore}

At first, we consider a pore arbitrarily chosen in the cubic of the membrane with unit length. Set cartesian coordinate, in which the original point is placed at the center of gravity of the pore and $X$ and $Y$ axes are taken in parallel to the membrane surface (and accordingly, $Z$ axis is perpendicular to the surface) (see Figure 2). Draw numerous lines parallel to the $X$ axis, some of which penetrate into the pore in question. The length of the straight line, cut off by ellipsoid, (i.e., the distance between two intersecting points of the line with two sides, faced each other; of a given ellipsoid as a pore surface) is expressed by $x$ (see Figure 2) and hereafter $x$ is referred to as simply the cut-off length. A set of nodes where the cut-off length is in the range of $x$ and $x+\mathrm{d} x$ is shown 


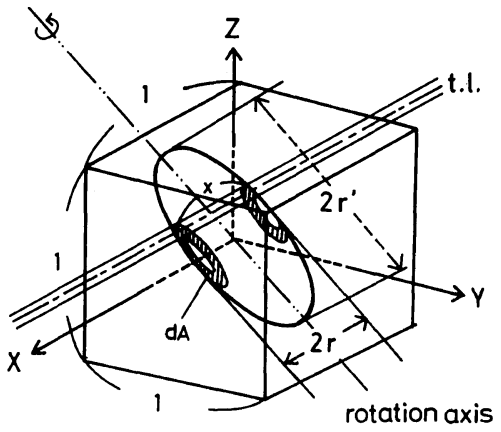

Figure 2. Ellipsoidal pore in a unit cube of the membrane: $r$, rotational radius; $r^{\prime}$, half length of major axis of the ellipsoid; $x$, the distance between two intersecting points of the line with the ellipsoid (cut-off length); $\mathrm{d} A$, a collection of the intersecting points of the lines whose cut-off length are in the range of $x$ and $x+\mathrm{d} x$.

as shadowed area between two concentric circles, (i.e., as "doughnut" shape) in the figure. A pair of "doughnut" coexists in one pore. Here, the area of a shadowed doughnut is expressed by $\mathrm{d} A$ and the radius of the pore is defined by the maximum value measured along the perpendicular direction to the rotational axis and denoted by $r$.

The ellipsoidal pore can be represented by eq 1

$$
\begin{gathered}
a x^{2}+b y^{2}+c z^{2}+2 f y z+2 g z x \\
+2 h x y+d=0
\end{gathered}
$$

where $a, b, c, d, f, g$, and $h$ are parameters characteristic of the pore shape. The probability, $P_{\mathrm{e}}(x, r) \mathrm{d} x$, that the cut-off length lies between $x$ and $x+\mathrm{d} x$, is linearly propotional to the shadowed area $\mathrm{d} A$. Since the length of the sides of the membrane in question is unity then we obtain

$$
P_{\mathrm{e}}(x, r) \mathrm{d} x=\mathrm{d} A
$$

$\mathrm{d} A$ in eq 2 is given on the basis of eq 1 by

$$
\mathrm{d} A=\left(\frac{\pi}{2}\right) \Phi_{\mathrm{e}} x \mathrm{~d} x
$$

with

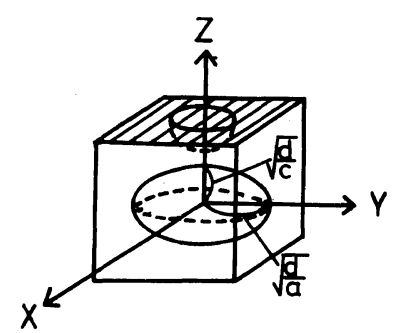

Figure 3. Ellipsoidal pore model in the case of membranes prepared by micro-phase separation method: $(d / a)^{1 / 2}$, rotational radius; $(d / c)^{1 / 2}$, radius perpendicular to the rotational axis.

$$
\Phi_{\mathrm{e}}=\frac{a^{2}}{\left(\left(h^{2}-a b\right)\left(g^{2}-a c\right)-(g h-a f)^{2}\right)^{1 / 2}}
$$

Combination of eq 2 and 3 yields

$$
P_{\mathrm{e}}(x, r) \mathrm{d} x=\frac{\pi}{2} \Phi_{\mathrm{e}} x \mathrm{~d} x
$$

Since $\Phi_{\mathrm{e}}$ is independent of $x, y$, and $z$ and only depends on the parameters characterizing the ellipsoid (i.e., $a, b, c, d, f, g$, and $h$ in eq 1), $\Phi_{\mathrm{e}}$ is called as a shape factor. In general, membranes prepared by the micro-phase separation process have the pores with rotating axis parallel to $Z$ axis, as demonstrated in Figure 3, because the phase separation occurs within a very thin layer parallel to the surface of a casting solution. ${ }^{10}$ Accordingly, in eq $1 a=b$ and $f=g=h=0$ hold. Equation 4 can be simplified to

$$
\Phi_{\mathrm{e}}=(a / c)^{1 / 2}
$$

Secondly, we consider the membrane consisting of many pores with different pore radius (i.e., $r$ in the ellipsoidal pore). The shape of pore, expressed by $\Phi_{\mathrm{e}}$, is assumed to be independent of $r$. The pore radius distribution function is defined as $N_{\mathrm{e}}(r) \mathrm{d} r$. Here, the suffix e means ellipsoidal pore model.

Consider a unit cubic body in the membrane. The number of the lines, parallel to $X$ axis, having the cut-off length of $x \sim x+\mathrm{d} x$ per unit volume is defined as the distribution of $x$, $F(x) \mathrm{d} x, F(x)$ is directly related to the pore radius distribution $N_{\mathrm{e}}(r)$ through the relation 


$$
F(x) \mathrm{d} x=\int_{x / 2}^{\infty} P_{\mathrm{e}}(x, r) N_{\mathrm{e}}(r) \mathrm{d} x \mathrm{~d} r
$$

Here, note that the lower limit of the integration of eq 7 is not zero, but $x / 2$, because for a given $x$ the pores with $2 r<x$ are not counted in the integral. Solving eq 7 for $N_{\mathrm{e}}(r)$ by using eq 5 , one obtains

$$
N_{\mathrm{e}}(r)=-\frac{4}{\pi \Phi_{\mathrm{e}}}\{\mathrm{d}(F(x) / x) / \mathrm{d} x\}
$$

By observing the electron microphotograph of the membrane we can determine the shape factor $\Phi_{\mathrm{e}}$, $\left(\right.$ i.e., $\left.(a / c)^{1 / 2}\right)$ and then using eq 8 , we can estimate $N_{\mathrm{e}}(r)$ for ellipsoidal pores from $F(x)$ determined experimentally from the electron microphotograph of a membrane.

\section{Spherical Pore}

A spherical pore is a specialized ellipsoidal pore with $a=c$ (i.e., $\left.\Phi_{\mathrm{e}}=1\right)$. In this case, eq 8 can be simplified as

$$
N_{\mathrm{s}}(r)=-\frac{4}{\pi}(\mathrm{d}(F(x) / x) / \mathrm{d} x)
$$

where, suffix s means sphere pore model and $N_{\mathrm{s}}(r)$ is pore radius $r$ distribution function.

\section{Straight-through Cylindrical Pore}

For cylindrical pores (i.e., straight-through cylindrical pore model shown on Figure 1(a)), $\Phi_{\mathrm{e}}$ is infinite and we cannot apply eq 8 to this kind of pores. Another approach starting from eq 1 should be adopted for cylindrical pore model.

Figure 4 shows the schematic representation of the cross sectional plane obtained by slicing along the parallel direction to the membrane surface. The observed shape of the pore on the surface is a circle. The plane is taken as a unit square, with sides parallel to $X$ and $Y$ axes respectively. At first, the case of one pore being in the unit square is employed and then the case of many pores is considered. The origin of a cartesian coordinate is set at the center of the circle. When we draw a test line with unit

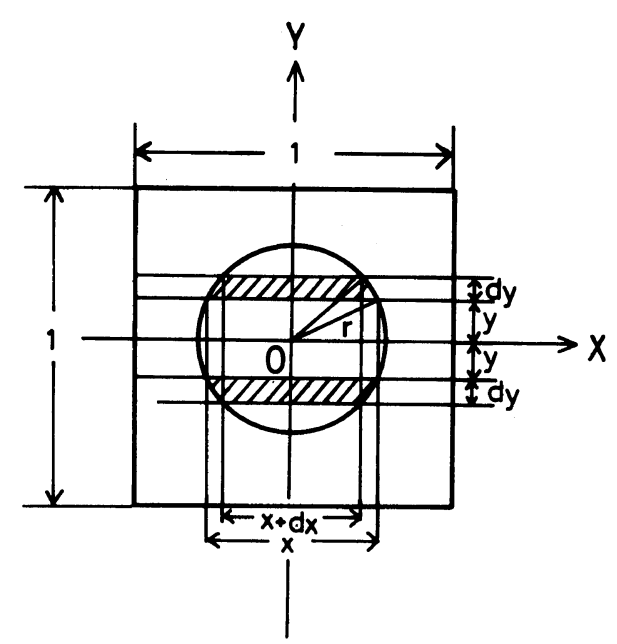

Figure 4. Schematic representation of the cross sectional plane in the case of straight-through cylindrical pores: $r$, radius of straight-through cylindrical pore; $x$, length of the test line which crosses the circular pores (cut-off length); $y$, distance between the center of the pore circle and test line.

length parallel to $X$ axis, the line crosses the circle representing a cylindrical pore with radius of $r$. The cut-off length is represented by $x$. The value of $x$ depends on the coordinate $y$ and the radius $r$. The set of the line whose cutoff length is between $x$ and $x+\mathrm{d} x$ is shown as shadowed area in Figure 4. The probability of finding the set of the lines with cut-off length $x$ for cylindrical pore with radius $r$ is denoted as $P(x, r) \mathrm{d} x$, where $P(x, r)$ is the probability density of $x$ for the pore with the radius $r$. $P(x, r) \mathrm{d} x$ equals to the length of the projection of the shadowed area in Figure 4 to the $Y$ axis. That is, eq 10 holds

$$
P(x, r) \mathrm{d} x=-2 \mathrm{~d} y
$$

The minus sign is attached in eq 10 by taking into account the positive direction of $X$ and $Y$ axes in Figure 4. As the shape of cross sectional view of straight-through cylindrical pore on the $X, Y$ plain is circular, eq 11 holds

$$
\left(\frac{x}{2}\right)^{2}+y^{2}=r^{2}
$$

By differentiating eq 11 with respect to $x$ we 
obtain

$$
\mathrm{d} y=\frac{-x \mathrm{~d} x}{2\left(4 r^{2}-x^{2}\right)^{1 / 2}}
$$

Substitution of eq 12 into eq 10 leads

$$
P(x, r)=\frac{x}{\left(4 r^{2}-x^{2}\right)^{1 / 2}}
$$

The number of pore whose radius is in the range $r \sim r+\mathrm{d} r$ in a unit area of a membrane is defined by $N(r) \mathrm{d} r .^{2}$ Here, $N(r)$ is pore radius distribution function. $F(x) \mathrm{d} x$ is given by eq 14 derived in the same procedure as eq 7

$$
F(x) \mathrm{d} x=\int_{x / 2}^{\infty} P(x, r) \mathrm{d} x N(r) \mathrm{d} r
$$

By substituting $P(x, r)$ of eq 13 into eq 14 , we obtain

$$
F(x)=x \int_{x / 2}^{\infty} \frac{N(r)}{\left(4 r^{2}-x^{2}\right)^{1 / 2}} \mathrm{~d} r
$$

As eq 15 cannot be solved analytically we can only evaluate $N(r)$ from $F(x)$ observed through the numerical calculus.

\section{EXPERIMENTAL}

\section{Sample}

\section{Porous Polymeric Membranes}

The porous cellulose acetate membranes were prepared, using a micro-phase separation method, from the solution of cellulose acetate (combined acetic acid of $54.1 \mathrm{wt} \%$ )/ $\mathrm{CaCl}_{2} \cdot 2 \mathrm{H}_{2} \mathrm{O} /$ acetone/methanol/cyclohexanol according to the method described in the previous paper. ${ }^{9}$ The porous regenerated cellulose membrane were prepared by a microphase separation method from the solution of cellulose cuprammonium solution/acetone. ${ }^{11}$ These membranes were used as the model membrane with pores of complicated shape.

A commercially available polycarbonate membrane Nuclepore ${ }^{\circledR}(\mathrm{Nu} 0.08)$ manufactured by General Electric Co. (U.S.A.) was used as a model membrane with straightthrough cylindrical pores.

\section{Swelling Agents}

Xylene, acetone, dimethylformamide (DMF), glycerin and water were used as swelling agent for evaluating the porosity by the swelling method. ${ }^{2}$ All of the chemical reagents were of guaranteed reagent grade manufactured by Kishida Chemical Company, Ltd. (Japan).

\section{Measurement}

1) Pore Radius Distribution and Mean Pore Radius

Electron Microscopic (EM) Method. Thin samples with ca. $0.05 \sim 1 \mu \mathrm{m}$ in thickness were prepared by slicing the original membrane solidified with acrylic resin and were observed under a scanning electron microscope (SEM, "JSM-U3" type, manufactured by Japan Electron Optics Laboratory Co., Ltd., Japan).

For the replica micrographs, pieces of the membrane were coated with platinum and then with carbon. The replica thus prepared was peeled off from the membrane piece by dissolving the membrane with $99 \%$ sulfuric acid solution. The replica were washed with water and observed by a transmission electron microscope (TEM, HU-11B type, manufactured by Hitachi Co., Ltd., Japan).

Mercury Intrusion (MI) Method. A mercury intrusion apparatus constructed in our laboratory $^{8}$ was employed. The volume contradiction of the sample and mercury, $\Delta V(p)$, corresponding to the loading pressure $p$, can be detected as the mercury pillar height of the dilatometer.

The pore radius distribution function $N(r)$ can be evaluated from the observed $\mathrm{d} \Delta V(p) /$ $\mathrm{d} P$ by

$$
r_{\mathrm{x}}=2 \sigma \cos \left(\theta+\theta_{\mathrm{p}}\right) / P
$$

$$
\begin{aligned}
N\left(r_{\mathrm{x}}+\frac{d}{2} \tan \theta_{\mathrm{R}}\right)= & \frac{-P^{4}}{8 \pi V_{\mathrm{s}} \sigma^{3} \cos ^{3}\left(\theta+\theta_{\mathrm{R}}\right)} \\
& \times\left\{\frac{\mathrm{d} \Delta V(p)}{\mathrm{d} P}-V_{\mathrm{g}} \beta_{\mathrm{g}}-\frac{V_{\mathrm{a}}}{\left(\beta_{\mathrm{g}}\right)^{2}}\right\}
\end{aligned}
$$


where, $r_{\mathrm{x}}$ is the pore radius at the position $x$ from the membrane surface, $V_{\mathrm{g}}$ the volume of mercury in the dilatometer, $V_{\mathrm{s}}$ the volume of specimen, $\sigma$ the interfacial tension of mercury, $\theta_{\mathrm{R}}$ the angle defined in Figure 1(b) for truncated cone shape pore. All parameters except for $\mathrm{d} \Delta V(p) / \mathrm{d} P$ in eq 17 can be determined in advance. Equation 17 is a general formula of the equation derived in the previous article (eq 10 in ref 2).

Fluid Permeability and Bubble Point (BP) Method. A membrane was dipped in ethanol prior to measurements to make sure the sufficient penetration of solvent into a pore in the membrane. The apparatus constructed in our laboratory ${ }^{8}$ was employed. By putting the flux of air $J$ as a function of loaded pressure $\Delta P$ into eq $18, N(r)$ for the straight-through cylindrical pore model can be calculated

$$
N(r)=\{\mathrm{d}(J / \bar{P}) / \mathrm{d} \ln \Delta P-J / \bar{P}\} /\left(2 \sigma k(2 \sigma / \Delta P)^{4}\right\}
$$

where, $k$ is given by $\pi / 8 \eta \mathrm{d}$ ( $\eta$, viscosity of the fluid; $d$, thickness of the membrane), and $\bar{P}$ is $(\Delta P+1)$ atm.

Flow Rate (FR) Method. ${ }^{2}$ The mean pore radius $r_{\mathrm{f}}$ was calculated by putting porosity $\mathrm{Pr}$ and the filtration ratio per unit area of a membrane $J$ into eq 19 derived under the assumption of straight-through cylindrical pore model.

$$
r_{\mathrm{f}}=\left\{\left(\frac{8 \eta J}{P_{\mathrm{r}}}\right)\left(\frac{d}{\Delta P}\right)\right\}^{1 / 2}
$$

where, $\eta$ is the viscosity of water, $d$ the thickness of the membrane, $\Delta P$ the loaded pressure and $r_{\mathrm{f}}$ corresponds to $\left(\bar{r}_{3} \cdot \bar{r}_{4}\right)^{1 / 2}$. Here, $\bar{r}_{3}$ and $\bar{r}_{4}$ are the third and the fourth mean pore radii respectively defined below in the case of the membrane with straight-through cylindircal pore.

$$
\bar{r}_{\mathrm{i}}=\int r^{i} N(r) / \int r^{i-1} N(r) \mathrm{d} r \quad(i=1,2,3, \cdots)
$$

Surface Area (SA) Method. By using the surface area analyzer (Micromeritics ${ }^{\circledR}$ manufactured by Shimadzu Seisakusho, Japan), the surface area of the membrane, $S r$ was evaluated by Brunauer, Emmett and Teller (BET) method. The mean pore radius $\bar{r}_{\mathrm{s}}$ can be evaluated from the experimental value of $\mathrm{Pr}$ and $S r$ using eq 21 derived on the basis of the straight-through cylindrical pore model

$$
\bar{r}_{\mathrm{s}}=2 \operatorname{Pr} / S r
$$

the relation of $\bar{r}_{\mathrm{s}}=\bar{r}_{2}$ holds for this pore model.

\section{2) Porosity $\mathrm{Pr}$}

The porosity obtained by the apparent density method $\operatorname{Pr} \rho$ was calculated by

$$
\operatorname{Pr} \rho=\left(1-\rho_{\mathrm{a}} / \rho_{\mathrm{p}}\right) \cdot 100(\%)
$$

where, $\rho_{\mathrm{p}}$ is the density of the material constructing the membrane and $\rho_{\mathrm{a}}$ is the apparent density of the membrane. When the membrane is immersed into the solvent with the density of $\rho_{\mathrm{s}}$, the solvent penetrates into the pore inside the membrane. The porosity obtained by the swelling method Prs can be calculated by measuring the weight of the membrane after immersion $W_{\mathrm{s}}$ and using eq 23.

$$
\operatorname{Prs}=\left(\frac{W_{\mathrm{s}}}{\rho_{\mathrm{s}}}\right) /\left(\frac{W_{\mathrm{p}}}{\rho_{\mathrm{p}}}+\frac{W_{\mathrm{s}}}{\rho_{\mathrm{s}}}\right)
$$

where $W_{\mathrm{p}}$ is the dry weight of the membrane and $W_{\mathrm{s}}$ is the weight of the membrane swelled with the solvent.

\section{ANALYTICAL PROCEDURE}

At first, by using the average value of the shape factor $\Phi_{\mathrm{e}}$ determined by electron microscopic observations for the two cross sections parallel and perpendicular to the membrane surface, we should determine the pore shape, i.e., spherical, ellipsoidal, or cylindrical pore. For the porous membrane prepared by microphase separation method, it is hard to determine the pore shape because of the complicated pore shape. In this case, the shape of 


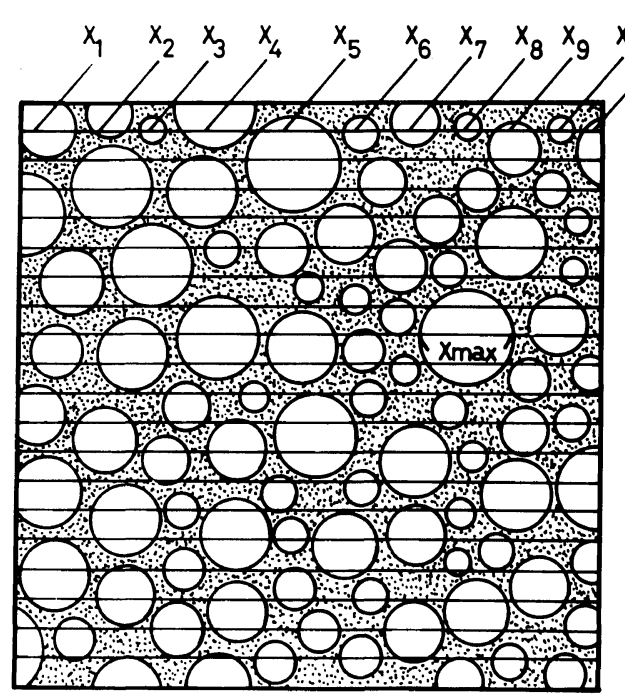

(a)

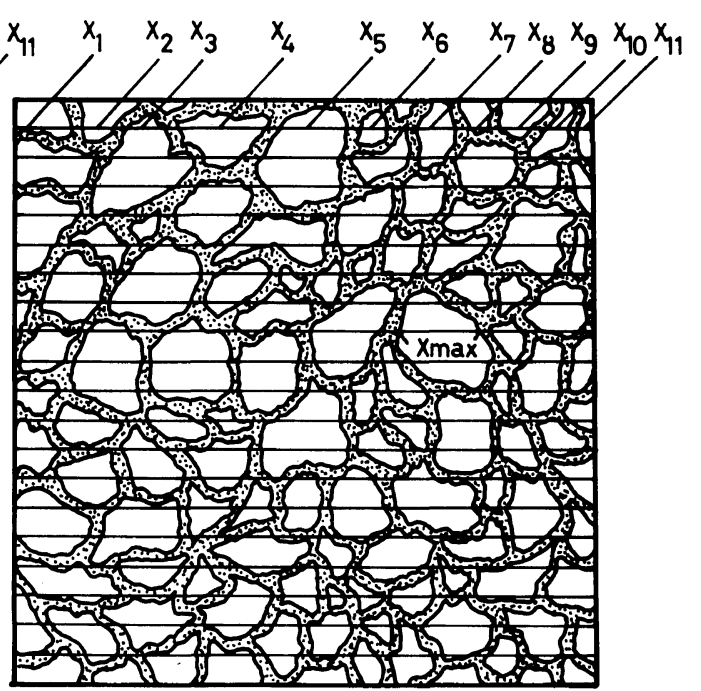

(b)

Figure 5. Schematic representation of an electron micrographs of the plane parallel to membrane surface. The vacant regions surrounded by dotted area indicate pores; (a), a membrane which has ellipsoidal pores; (b), an actual membrane which has complicated pores; $x_{1}, x_{2}, x_{3}, \cdots, x_{11}$, and $x_{\max }$ indicate cut-off length.

pores on the plane parallel to the membrane surface is assumed to be circular. Then the analytical procedure for ellipsoidal pore should be employed since ellipsoidal shape is general case of spherical shape. The average value of the ratio of $(a / c)^{1 / 2}$ (i.e., $\Phi_{\mathrm{e}}$ in this case) is determined through the electron micrograph of the cross section perpendicular to the membrane surface.

On the electron micrographs of the plane parallel to the membrane surface taken with the magnitude adequate for scaling the cut-off length $x$ for the membrane, some ten test lines, which are parallel to each other are drawn. The space of the test line is less than the average diameter of the pore so as that the number of test lines that cross a pore is more than one.

Figure 5(a) shows schematic representation of an electron micrograph of the membrane with ellipsoidal pores. On this photograph, 20 test lines are drawn. The cut-off length $x_{1}, x_{2}$, $x_{3}, \cdots$ are measured. The maximam of cut-off length $x_{\max }$ is determined. The membrane pre- pared by micro-phase separation method has complicated shape pore as shown in Figure 5(b). Even in this case, the cut-off length $x_{1}, x_{2}$, $x_{3}, \cdots$ and $x_{\max }$ are measured in similar manner to the case of Figure 5(a). The interval of $x, \Delta x$, can be defined as $\Delta x=x_{\max } / I$, where $I$ is decided under taking into consideration the accuracy of $F(x)$ to be calculated. And the total number of data of cut-off length is 50 in general case. The data of $x$ is classified in one of the range of $0 \sim \Delta x, \Delta x \sim 2 \Delta x, 2 \Delta x \sim 3 \Delta x$, $\cdots$ and so on. We get the number of data $\left[F_{x}\right]$ belonging to each range. $\left[\mathrm{F}_{\mathrm{x}}\right]$ reflects $F(x)$ by the following equation of $F(x)=\left[\mathrm{F}_{\mathrm{x}}\right] / \Delta x$. The minor modification of $F(x)$ is carried out by representing $F(x)$ vs. $x$ curve with smooth curve if necessary. By putting this $F(x)$ into eq 8,9 , and $15, N_{\mathrm{e}}(r), N_{\mathrm{s}}(r)$, or $N(r)$ corresponding for various pore models can be calculated.

For instance, by using $N_{\mathrm{e}}(r)$ thus obtained, we can calculate porosity $P_{\mathrm{e}} r$, pore density $N_{\mathrm{e}}$, and $i$-th mean pore radii $\bar{r}_{\mathrm{e} i}(i=1,2,3,4)$ are calculating with the use of the following equations 


$$
\begin{gathered}
P_{\mathrm{e}} r=\pi \int r^{2} N_{\mathrm{e}}(r) \mathrm{d} r \\
N_{\mathrm{e}}=\int N_{\mathrm{e}}(r) \mathrm{d} r \\
\bar{r}_{\mathrm{e} 1}=\int r N_{\mathrm{e}}(r) \mathrm{d} r / N_{\mathrm{e}} \\
\bar{r}_{\mathrm{e} 2}=\int r^{2} N_{\mathrm{e}}(r) \mathrm{d} r / \int r N_{\mathrm{e}}(r) \mathrm{d} r \\
\bar{r}_{\mathrm{e} 3}=\int r^{3} N_{\mathrm{e}}(r) \mathrm{d} r / \int r^{2} N_{\mathrm{e}}(r) \mathrm{d} r \\
\bar{r}_{\mathrm{e} 4}=\int r^{4} N_{\mathrm{e}}(r) \mathrm{d} r / \int r^{3} N_{\mathrm{e}}(r) \mathrm{d} r
\end{gathered}
$$

The ratio of $\bar{r}_{\mathrm{e} i} / \bar{r}_{\mathrm{e} i-1}$ reflects the width of the pore size distribution and we chose $i=4$ in this paper. These eq $24 \sim 29$ correspond to eq 20 in the case of straight-through cylindrical pore.

\section{RESULTS AND DISCUSSION}

\section{Pore Radius Distribution}

Figure 6(a), (b) shows the electron micrographs of the back surface and the cross section perpendicular to this surface of the regenerated cellulose membrane. The dark regions indicate pores. The shape of the pores for this membrane is not circular. We must judge what these pores should be classified into. It is clear from this figure that these pores are far from the straight-through cylindrical pores. Then we chose ellipsoidal and spherical pore models.

First, we compare $N_{\mathrm{e}}(r)$ for ellipsoidal pore model with $N_{\mathrm{s}}(r)$ for spherical pore model obtained for regenerated cellulose membrane. The result is shown in Figure 7(a). Here, the front surface (curve 1) is the surface from which the solvent in casting solution vaporized during membrane forming process and the back surface (curve 2 ) is the surface opposite to the front surface. The observed value of $\Phi_{\mathrm{e}}$ evaluated from Figure 6(b) was 0.32 , indicating that the ellipsoidal pore model was more suitable than spherical pore model in order to represent the regenerated cellulose membrane. $N_{\mathrm{s}}(r)$ curve coincides with $N_{\mathrm{e}}(r)$ curve if the $N_{\mathrm{e}}(r)$ curve is shifted by $\log \Phi_{\mathrm{e}}$ parallel along vertical axis to the positive direction. The front surface has a larger mean pore radius and broader pore radius distribution than the back surface in both models of ellipsoidal and spherical pores.

Secondly, we compare various $N(r)$ obtained by EM, MI, and BP methods assuming straight-through cylindrical pore model, though this assumption is not allowable in the

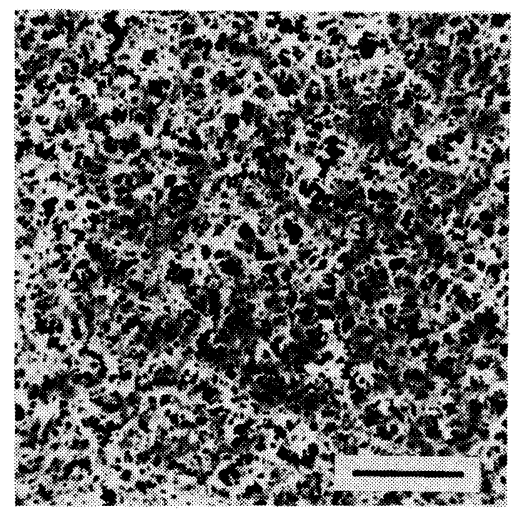

(a)

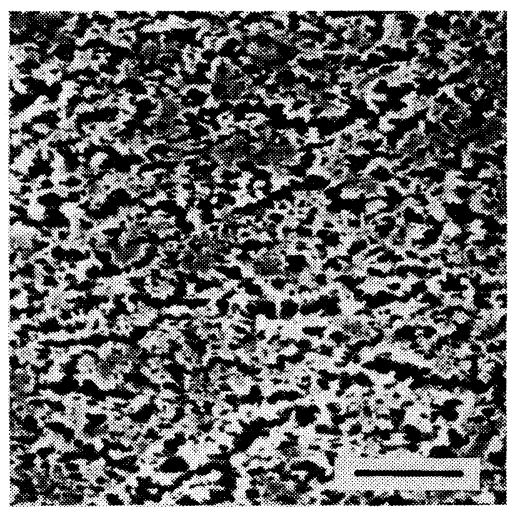

(b)

Figure 6. Electron micrographs of the regenerated cellulose membrane: (a), the back surface; (b), the cross section perpendicular to the surface of a membrane; a scale bar indicates $5 \mu \mathrm{m}$. 

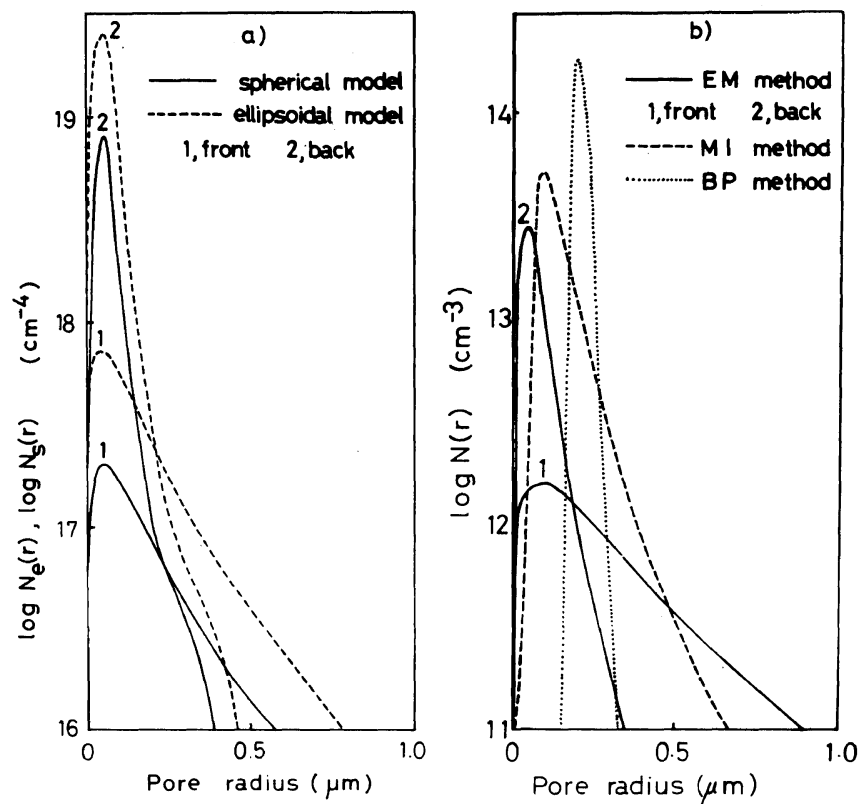

Figure 7. Pore radius distribution of regenerated cellulose membrane by various methods: 7(a), spherical pore model by EM; ----, ellipsoidal pore model by EM; 1, front surface; 2, back surface; 7(b), straight-through cylindrical pore model; __ , by EM method; 1, front surface; 2, back surface; --_--, by MI method; $\cdots$, by BP method.

strict sense. Figure 7(b) shows the results. EM method affords us two $N(r)$ of the front and back surfaces. All $N(r)$ cover similar $r$ range, but the absolute value of $N(r)$ varies depending of the methods employed. BP method gives the most sharp $N(r)$ among three methods. The broadness of $N(r)$ curve obtained by $\mathrm{MI}$ method is just between those of front and back surfaces of the membrane by EM method. The difference in these four $N(r)$ curves may be originated from the difference in the minimum pore radius countable as a pore in various methods, and also the difference in the pore shape. When the actual minimum pore radius of the membrane is larger than the minimum pore radius countable for the various methods and when the pore shape is definitely determined as straight-through cylindrical pore as in the case of Nuclepore, ${ }^{\circledR}$ the difference in $N(r)-r$ curves obtained by the various methods are negligibly small. ${ }^{2}$

Figure 8 shows $N_{\mathrm{s}}(r)$ estimated by EM method for regenerated cellulose membranes with different mean pore radius prepared by micro-phase separation method. When the mean pore radius increases the peak value of $N_{\mathrm{e}}(r)$ decreases and the slope of $N_{\mathrm{e}}(r)$ vs. $r$ curve at larger $r$ region becomes smaller. Neither $N_{\mathrm{e}}(r)$ nor $N_{\mathrm{s}}(r)$ could be represented by Poisson's distribution or most probable distribution. However, $N_{\mathrm{e}}(r)$ shown in Figure 8 is well reproducible by the theory of pore formation, proposed previously. ${ }^{10}$

\section{Pore Characteristics Evaluated by Various Methods}

Table I summarizes some pore characteristics of a regenerated cellulose membrane and a polycarbonate membrane Nuclepore. ${ }^{\circledR}$ The porosity and also the $i$-th mean pore radii $\bar{r}_{i}$ evaluated by EM method coincides with those by the various other methods for the membrane of Nuclepore ${ }^{\circledR}$ which is ascertained to be representable by straight-through cylindrical 


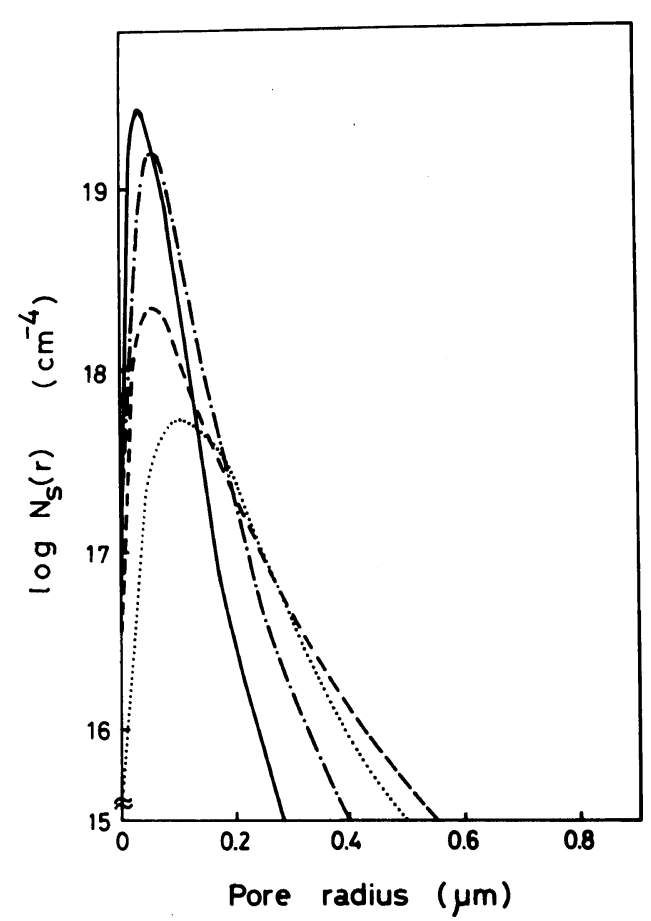

Figure 8. Pore radius distribution curves $N_{\mathrm{s}}(r)$ for regenerated cellulose membranes with different mean pore radius prepared by micro-phase separation method: $\longrightarrow,\left(\bar{r}_{3} \cdot \bar{r}_{4}\right)^{1 / 2}=0.076(\mu \mathrm{m}) ;-\cdot-,\left(\bar{r}_{3} \cdot \bar{r}_{4}\right)^{1 / 2}=0.13$ $(\mu \mathrm{m}) ;----,\left(\bar{r}_{3} \cdot \bar{r}_{4}\right)^{1 / 2}=0.32(\mu \mathrm{m}) ; \cdots,\left(\bar{r}_{3} \cdot \bar{r}_{4}\right)^{1 / 2}=0.24$ $(\mu \mathrm{m})$. pore model. Therefore, we can confirm the reliability of the numerical difference calculus method in order to solve eq 15 for EM method.

The mean pore radii of the back surface of regenerated cellulose membrane, evaluated by EM method, are near to those obtained by FR and BP methods. The mean pore radii obtained by MI method is experimetally similar to the average value of those of front and back surfaces in EM method. That is, all the radii evaluated by various methods distribute between those of front and back surfaces evaluated by EM method. By putting $N(r)$ and $N_{\mathrm{e}}(r)$ thus obtained into eq 20 and eq 26-29, respectively, we can conclude that $\bar{r}_{i}$ is larger than $\bar{r}_{\mathrm{e} i}$ by EM method when $i$ is less than 4 and the dependence of $\bar{r}_{i}$ on $i$ is less than that of $\bar{r}_{\mathrm{e} i}$.

The porosity $\mathrm{Pr}$ of the regenerated cellulose membrane prepared by micro-phase separation is affected by the methods employed for evaluation as shown in Table $\mathrm{I}$. This is because all of the pores do not contribute to the porosity. For example, in MI method, only open pores contribute to the porosity, but isolated pores do not. The apparent density

Table I. Pore characteristics of membranes evaluated by various methods

\begin{tabular}{|c|c|c|c|c|c|c|c|c|c|c|}
\hline \multirow{2}{*}{ Membrane } & \multirow{2}{*}{\multicolumn{2}{|c|}{$\begin{array}{l}\text { Evaluation } \\
\text { methods }\end{array}$}} & \multirow{2}{*}{$\frac{\bar{r}_{1}}{\mu \mathrm{m}}$} & \multirow{2}{*}{$\frac{\bar{r}_{2}}{\mu \mathrm{m}}$} & \multirow{2}{*}{$\frac{\bar{r}_{3}}{\mu \mathrm{m}}$} & \multirow{2}{*}{$\frac{\bar{r}_{4}}{\mu \mathrm{m}}$} & \multirow{2}{*}{$\frac{\left(\bar{r}_{3} \cdot \bar{r}_{4}\right)^{1 / 2}}{\mu \mathrm{m}}$} & \multirow{2}{*}{$\frac{\bar{r}_{4} / \bar{r}_{3}}{-}$} & \multirow{2}{*}{$\frac{P r}{\%}$} & \multirow{2}{*}{$\frac{N}{\text { Number } / \mathrm{cm}^{2}}$} \\
\hline & & & & & & & & & & \\
\hline \multirow{8}{*}{$\begin{array}{l}\text { Regenerated } \\
\text { cellulose } \\
\text { membrane }\end{array}$} & \multirow{2}{*}{\multicolumn{2}{|c|}{$\begin{array}{ll}\text { EM } & \begin{array}{l}\text { Front } \\
\text { Back }\end{array}\end{array}$}} & 0.30 & 0.50 & 0.67 & 0.81 & 0.74 & 1.21 & 27.0 & $5.9 \times 10^{7}$ \\
\hline & & & 0.07 & 0.09 & 0.12 & 0.16 & 0.14 & 1.25 & 4.3 & $2.1 \times 10^{8}$ \\
\hline & \multicolumn{2}{|c|}{ MI } & 0.14 & 0.22 & 0.41 & 0.84 & 0.59 & 2.04 & 56.0 & $5.7 \times 10^{8}$ \\
\hline & \multicolumn{2}{|l|}{ BP } & 0.21 & 0.21 & 0.21 & 0.21 & 0.21 & 1.00 & 9.5 & $6.0 \times 10^{8}$ \\
\hline & \multicolumn{2}{|l|}{ FP } & - & - & - & - & 0.20 & - & - & - \\
\hline & \multicolumn{2}{|c|}{ App. density } & - & - & - & - & - & - & 71.9 & - \\
\hline & \multicolumn{2}{|c|}{ Swelling } & - & - & - & - & - & - & $\begin{array}{c}71.0 \\
73.7\end{array}$ & - \\
\hline & \multicolumn{2}{|l|}{ SA } & - & 0.37 & - & - & - & - & 90.3 & - \\
\hline \multirow{5}{*}{$\begin{array}{l}\text { Polycarbonate } \\
\text { membrane } \\
\text { "Nuclepore"® } \\
N u 0.8\end{array}$} & \multicolumn{2}{|l|}{ EM } & 0.36 & 0.46 & 0.50 & 0.57 & 0.53 & 1.13 & 21.0 & $5.4 \times 10^{7}$ \\
\hline & \multicolumn{2}{|l|}{ MI } & 0.31 & 0.32 & 0.33 & 0.35 & 0.34 & 1.02 & 18.0 & $5.5 \times 10^{7}$ \\
\hline & \multicolumn{2}{|l|}{ FP } & - & - & - & - & 0.53 & - & - & - \\
\hline & \multirow{2}{*}{\multicolumn{2}{|c|}{ Appl. density }} & - & - & - & - & - & - & 22.0 & - \\
\hline & & & - & - & - & - & - & - & 16.0 & - \\
\hline
\end{tabular}


and swelling methods can count all pores independent of their shape. On the other hand, in BP method, only open pores contribute to the porosity, resulting in the smallest values of porosity, except for the values of the back surface of the membrane by EM.

These features of the regenerated cellulose membrane are maintained also in porous cellulose acetate membranes prepared by microphase separation method. The shapes of the pore radius distribution curves of these cellulose derivative membranes are similar with each other, indicating that the pore radius distribution curves for any porous membrane prepared by the micro-phase separation method have the common feature as indicated by the theory proposed by us. ${ }^{10}$

\section{REFERENCES}

1. For example, W. Pusch and A. Walch, J. Membrane
Sci., 10, 325 (1982).

2. K. Kamide and S. Manabe, "Ultrafiltration Membrane and Application," A. R. Copper Ed., Plenum Publishing Corp., New York, N.Y., 1980, p 173.

3. K. Kamide, S. Manabe, and S. Kajita, unpublished work.

4. For example, A. Kakuta, M. Kuramoto, M. Ohno, H. Kushida, A. Tanioka, and K. Ishikawa, J. Polym. Sci., 18, 3229 (1980).

5. U. Merin and M. Cheryan, J. Appl. Polym. Sci., 25, 2139 (1980).

6. K. Mitteilungen, Acta Polymerica, 32, 726 (1981).

7. For example, N. Suwa, "Stereology," Iwanami Publishing Corp., Tokyo, 1977.

8. K. Kamide, S. Manabe, and T. Matsui, Kobunshi Ronbunshu, 34, 299 (1977).

9. K. Kamide, S. Manabe, T. Matsui, S. Kajita, and T. Sakamoto, Kobunshi Ronbunshu, 34, 205 (1977).

10. K. Kamide and S. Manabe, ACS Symposium Series, No. 269, "Materials Science of Synthetic Membranes," D. R. Lioyd, Ed., 1985, p 197.

11. S. Manabe, M. Iwata, and T. Inoue, Japanese Patent Application 58-89625 (1983). 